\title{
Fabrication Process of Microsurgical Tools for Single-Cell Trapping and Intracytoplasmic Injection
}

\author{
Pak Kin Wong, Umberto Ulmanella, and Chih-Ming Ho, Member, IEEE
}

\begin{abstract}
In this paper, we introduce chemical etching-based processes for fabricating micromanipulators from fused silica microcapillary tubing. The resulting devices are capable of manipulating individual cells and can be used for intracytoplasmic injection. Two types of micromanipulating tools: single-cell trappers and micro-injectors, were fabricated with two single-step etching processes. These etching mechanisms, which are either surface tension controlled or diffusion rate limited, are discussed in detail with experimental verifications. The fabrication processes are reproducible and amenable to mass production due to their simplicity and low fabrication cost. Single cell capture and intracytoplasmic injection have been successfully demonstrated on brassica oleracea protoplasts.

[1188]
\end{abstract}

Index Terms-Cell trapping, intracytoplasmic injection, micromanipulator, photobleaching.

\section{INTRODUCTION}

$\mathbf{M}$ EMS technology enables the fabrication of microdevices in the same length scale of various bioparticles, such as cells and macromolecules, providing tools for direct manipulation of such objects. Kim et al. [1] first reported a surface micromachined electromechanical gripper that can hold a single euglena cell in air. More recently, the operation of pneumatic and thermally driven cell cages has been demonstrated in aqueous media [2], [3]. Tools designed for biological manipulation should be effective in liquid medium, since cells and biomolecules naturally exist in an aqueous environment. The ambient liquid environment lends itself to the use of hydrodynamic forces for this purpose [4], [5].

Micromanipulators combined with precise fluidic and motion control have proven to be invaluable tools in transgenesis [6] and in vitro fertilization [7]. Fabrication techniques for these micromanipulators are crucial for practical viability. Currently, micromanipulators are produced by a "heating and pulling" method. A small region of the capillary tubing is heated with a laser or a filament and then pulled until the capillary tubing breaks, leaving a sharp tip. However, this process requires high cost micropipette pullers with sophisticated temperature and pulling speed control to ensure a reproducible geometry. Moreover, pipette pulling

Manuscript received October 28, 2003; revised April 17, 2004. Subject Editor A. J. Ricco. This work is supported by CMISE, a NASA supported Institute for bio-nano-information fusion, and DARPA bioflips program. This paper was based on the work presented at the MEMS Conference 2003, Kyoto, Japan.

P. K. Wong and C.-M. Ho are with the Mechanical and Aerospace Engineering Department, University of California, Los Angeles, CA 90095 USA (e-mail: pak@ucla.edu; chihming@ucla.edu).

U. Ulmanella is with the Department of Biomedical Engineering, University of California, Los Angeles, CA 90095 USA (e-mail: umberto@seas.ucla.edu). Digital Object Identifier 10.1109/JMEMS.2004.838382

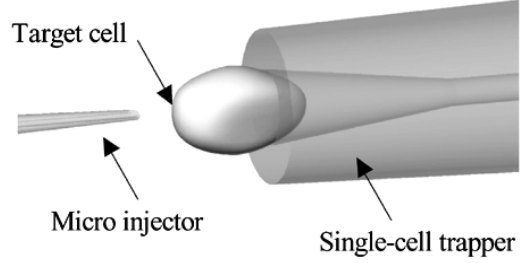

Fig. 1. Schematic of intracytoplasmic injection.

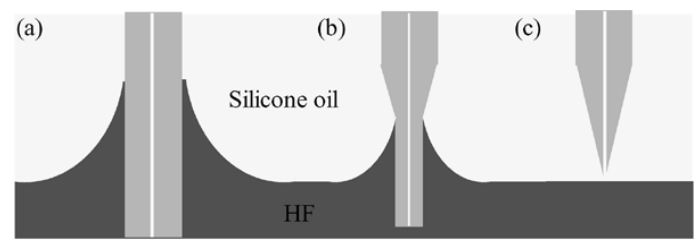

Fig. 2. Schematic illustrating the surface tension controlled sharpening process.

is a serial fabrication process difficult to apply to large-scale production.

In this paper, we present manufacturing processes based on chemical etching techniques to fabricate micromanipulators from fused silica micro capillary tubing. The wide range of dimensions for commercially available silica capillary tubing allows fabrication of microsurgical tools for various biological applications, such as transgenesis, patch clamp recording, zona drilling, and embryo biopsy. The processes we developed also offers distinct advantages to the heating and pulling method. Only one simple etching step is required to fabricate the various micromanipulators described in this study. Different dimensions and geometries of the devices can be obtained by modifying the etching conditions. The simplicity and reproducibility of the chemical etching processes can be easily adopted for low-cost, large-scale production.

We demonstrate the usefulness of micromanipulators by performing single cell capture and intracytoplasmic injection. Fig. 1 shows a schematic of the experiment. First, a cell trapper captures a target cell. Then, a sharpened microinjector directly penetrates the cell membrane and delivers desired fluid and embedded molecules to the target cell. In this report, the physical principles of the fabrication processes are presented with experimental verification. We first illustrate a surface tension controlled etching method for sharpening microcapillaries. Then, the fabrication of single-cell trappers via a diffusion limited etching method is discussed in detail. Microinjectors with highly tapered tips can also be fabricated with this method. 

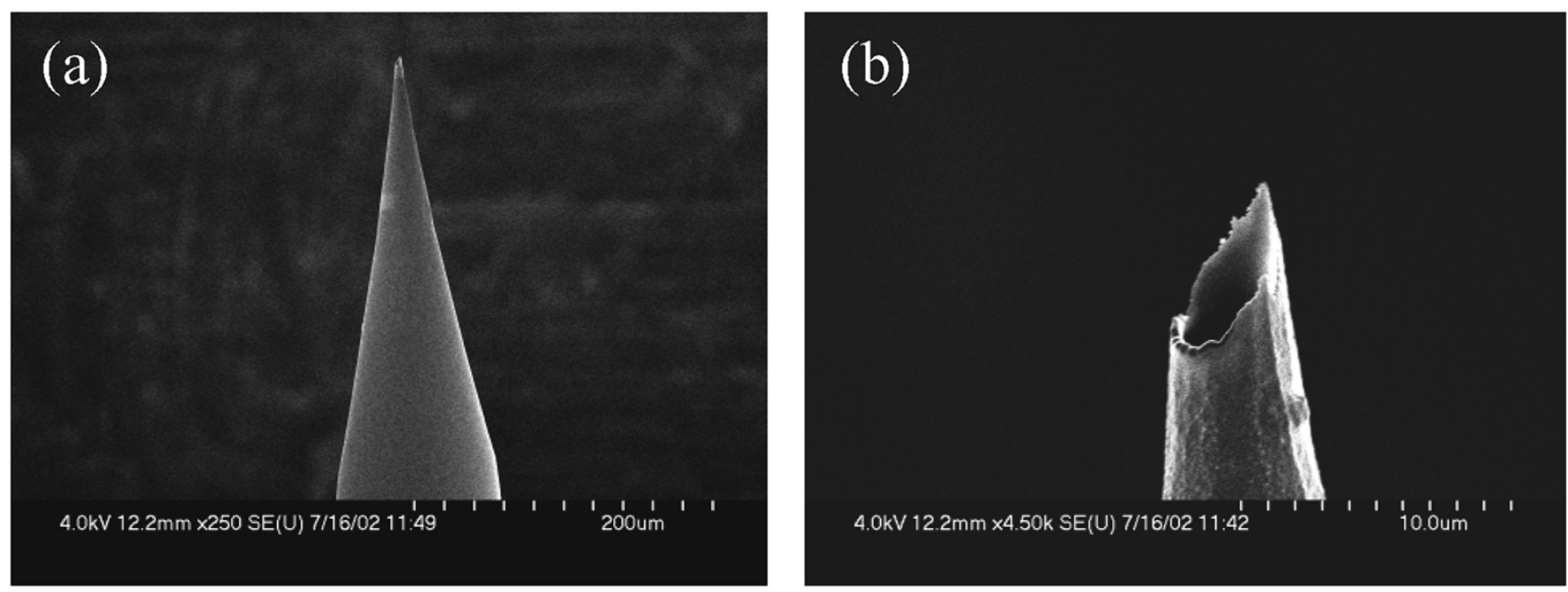

Fig. 3. SEM micrographs of (a) a sharpened capillary with roughly $2 \mu \mathrm{m}$ inner diameter, and (b) tip of the microinjector.

The application of our microsurgical devices results in the successful intracytoplasmic injection of fluorescent molecules into single brassica oleracea (cabbage) protoplasts, allowing photobleaching studies of intracellular physiological conditions.

\section{Microinjector Sharpened by Surface Tension CONTROLLED ETCHING}

Microinjectors can be fabricated with a chemical etching technique originally developed for sharpening optical fiber tips used in scanning near-field optical microscopy [8]. The chemical sharpening process utilizes surface tension force. Fig. 2 shows a schematic of this process. The capillary is immersed into an etchant bath with a layer of organic solvent atop the etchant. The etchant wets the surface of the capillary. The surface tension force is reduced as the outer diameter of the capillary decreases during etching. As a consequence, the height of the meniscus decreases with time until the etching front reaches the center of the capillary, forming a sharp tip. The force balance between the two opposing forces, i.e., surface tension and weight of the etchant, determines the final tip geometry. The profile of the meniscus can be described by solving the Young-Laplace equation in the axisymmetric case with the appropriate boundary conditions. We have performed independent experiments for measuring the surface tension and the contact angle to verify the etching model [9]. Experimental results agree with our etching model. We have also demonstrated a parallel-etching technique to control the tip geometry [9]. Tip angle can be adjusted from $7^{\circ}$ to $22^{\circ}$ by controlling the boundary condition of the meniscus.

The microinjectors are fabricated by etching $5-\mathrm{cm}$ long cut sections of fused silica capillary tubing with various inner diameters (Polymicro, TSP series). The approximate outer diameter of the capillaries is either 150 or $365 \mu \mathrm{m}$, which includes a respective $12-$ and $20-\mu \mathrm{m}$-thick polyimide coating. Roughly $2 \mathrm{~cm}$ of the polyimide coating are removed from one end of the capillaries, either thermally with an open flame, or chemically with hot piranha. Before etching, the tubing is filled with silicone oil by immersing the same end into the solvent and allowing the capillary forces to draw the oil inside. This step assures that the inner surface of the capillaries is protected from the etchant during the subsequent etching processes, thus preserving the inner geometry. The oil-filled capillary is then partially immersed into an HF solution (49\%) with a layer of silicone oil atop the solution. Surface tension causes the etchant to partially displace the silicon oil from inside the capillary. In order to ensure the fabrication of the sharp tip, the capillary is immersed to a depth such that the level of the HF/oil interface inside the capillary is much lower than the contact line between the etchant and capillary. With this precaution, the HF will etch a portion of the capillary from the inside out. The remaining portion of the capillary will be filled with oil, which preserves the inner geometry during the etching process. All the etching processes are performed at room temperature. The etching rate of fused silica in $49 \% \mathrm{HF}$ can be estimated from measuring the end diameters of the etched section of the capillaries at different times. Accurate determination of the etching rate is useful to prevent overetching of the inner geometry. The etch rate is $1.50 \mu \mathrm{m} \mathrm{min}^{-1}$ for our conditions. The chemical etching process is highly repeatable and has $100 \%$ overall yield. Fig. 3 shows SEM micrographs of a sharpened injector with a $\sim 2 \mu \mathrm{m}$ inner diameter.

\section{DIFFUSION LIMITED ETCHING OF TRAPPER AND INJECTOR}

\section{A. Single-Cell Trapper}

A single-cell trapper with a conical inner geometry can be fabricated by chemical etching in a diffusion-rate limited regime. The fabrication process, shown in Fig. 4, employs the same fused silica tubing used for the injectors. Unlike the previous process, the polyimide coating is not removed, as it serves to protect the outer geometry of the capillaries. In addition, the capillaries are carefully cleaved so that the end is flat and perpendicular to the axis of the capillary. First, the capillary is filled with silicon oil by capillary force. During the etching process, surface tension causes the etchant to partially displace the silicon oil from inside the capillary. Hydrofluoric acid is consumed during the chemical reaction and is replenished 


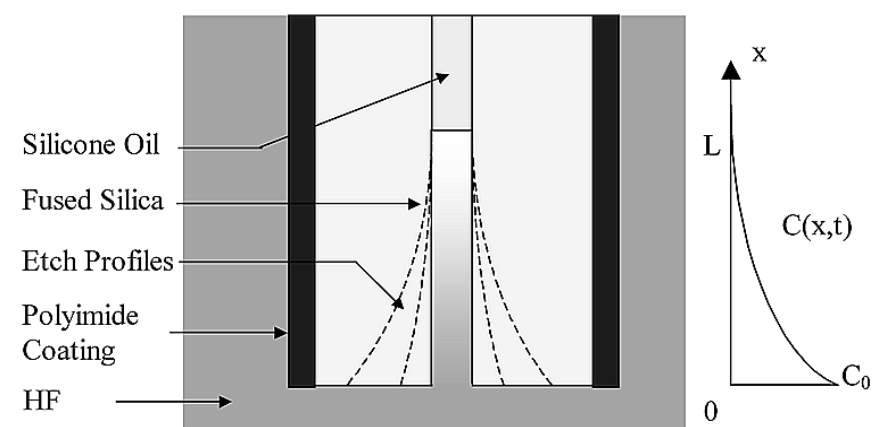

Fig. 4. Schematic of trapper etching with etchant concentration distribution inside the capillary.

by diffusion into the capillary. Therefore, the concentration distribution of the etchant inside the capillary is determined by both the diffusivity of the molecules and the kinetics of the chemical reaction. The resultant concentration and etching rate decrease with distance from the immersed end of the capillary.

To model the trapper formation, we assume that the process is one-dimensional axial symmetric, which can be justified by the high aspect ratio of the inner geometry of the capillary undergoing etching. Typically, the inner diameter of the capillary is less than $100 \mu \mathrm{m}$, while the length of the inner surface immersed into the etchant is on the order of centimeters. Using the coordinate system defined in Fig. 4, the diffusion equation that describes etchant concentration is given by (1). The initial and boundary conditions are given in (2) through (4).

$$
\begin{aligned}
\text { P.D.E. : } \frac{\partial C(x, t)}{\partial t} & =D \frac{\partial^{2} C(x, t)}{\partial x^{2}}-k C(x, t) \\
\text { I.C. }: C(x, 0) & =C_{0} \\
\text { B.C. }: C(0, t) & =C_{0} \\
\left.\frac{d C}{d x}\right|_{x=L} & =0
\end{aligned}
$$

where $C(x, t)$ is the concentration distribution at time $t, L$ is the initial meniscus height, $D$ is the diffusion constant, and $k$ is the first-order reaction rate coefficient. The two boundary conditions are derived from the bulk HF concentration, $C_{0}$, at the end of the capillary and the assumption that the diffusion rate at the oil-etchant interface is negligible. Since the volume inside the capillary is small, most of the etching is contributed from HF molecules diffusing from the bulk solution. The transient concentration distribution has minimal effect on the final trapper geometry. Therefore, we further simplify the model by considering only the steady-state concentration distribution of the etchant. Equation (5) shows the steady state solution of the etchant concentration distribution. For first order reaction kinetics, the etch profiles at different times can be estimated by multiplying the concentration distribution by the etching rate coefficient and the etching time

$$
C(x)=C_{0}\left(\cosh \frac{x}{\lambda}-\tanh \frac{L}{\lambda} \sinh \frac{x}{\lambda}\right)
$$

where $\lambda=(D / k)^{0.5}$ is the effective diffusion length. The length scale describes the average distance that molecules diffuse before being consumed in the chemical reaction. For $L \gg \lambda$, the

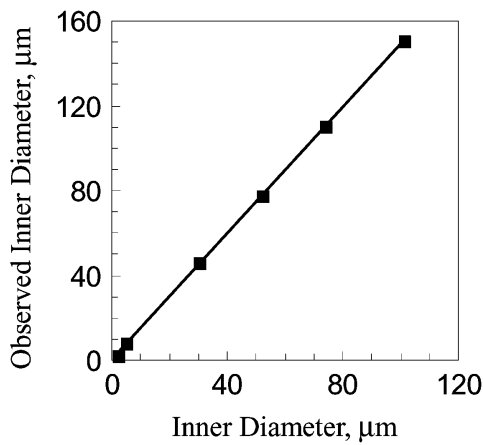

Fig. 5. Calibration of optical distortions.

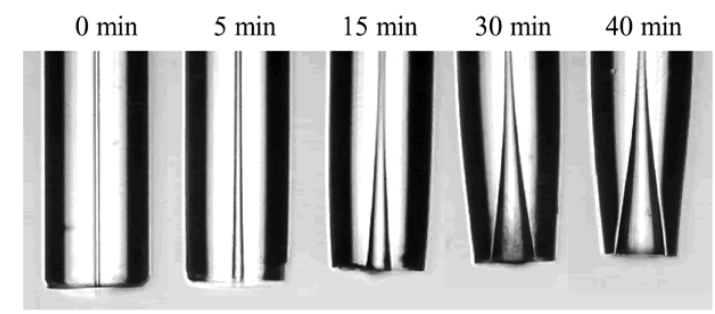

Fig. 6. Capillaries at different etching times during single-cell trapper formation. The outer diameter of the capillary before etching is $325 \mu \mathrm{m}$. The polyimide coating was removed after the etching process to take the pictures.

term $\tanh (L / \lambda)$ approaches 1 , and the steady state concentration distribution is approximately independent of the meniscus height $L$. Physically, this indicates that the diffusing molecules are rapidly consumed in the chemical reaction and the concentration of HF becomes negligible before reaching the etchant-oil interface.

A typical etching process requires less than thirty minutes depending on the desired dimensions of the trapper. The cylindrical geometry of the capillary and the difference in refractive indexes between the capillary and air cause the inner dimension of the cell trapper to appear larger than its actual size. This magnification can be determined by construction of a corresponding ray diagram [10] and is simply given by the ratio of the refractive indexes under the small angle approximation. We have experimentally calibrated the magnification by using capillaries with known inner diameters. For microcapillaries with a $325-\mu \mathrm{m}$-outer diameter, the small angle approximation is valid for up to a 100- $\mu \mathrm{m}$-inner diameter, as shown in Fig. 5. The magnification factor is found to be 1.48 , which is in excellent agreement with the refractive index of fused silica for wavelengths from $250 \mathrm{~nm}$ to $600 \mathrm{~nm}(1.50-1.45)$. Therefore, the actual inner geometry of the trapper can be estimated by simply using a correction factor.

Fig. 6 shows a series of capillaries after different etching times. The inner diameter of the conical region increases with time. It should be noted that etching can also occur at the lower end of the capillary which causes sharpening of the outer geometry. The mechanism of this process will be discussed in the next section. Fig. 7 shows the experimentally determined trapper geometries at different etching times compared with the diffusion model. The effective diffusion length is experimentally determined to be $169 \mu \mathrm{m}$. To verify the diffusion model, we obtained the diffusion constant of $\mathrm{HF}\left(1.6 \times 10^{-5} \mathrm{~cm}^{2} \mathrm{~s}^{-1}\right)$ 


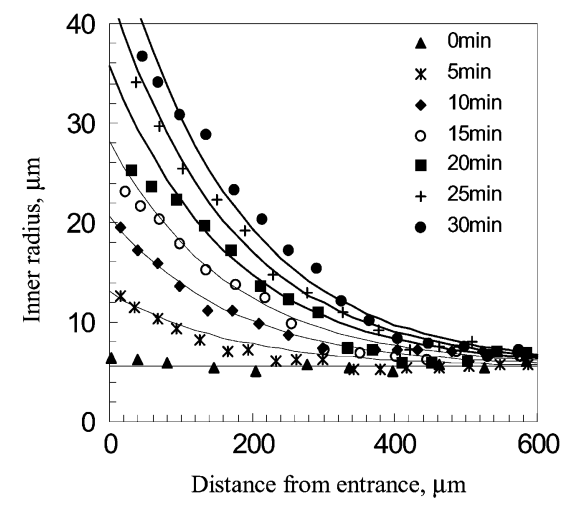

Fig. 7. Profiles of the single-cell trapper as a function of etching time. Solid lines represent the etched profiles calculated with the diffusion model.

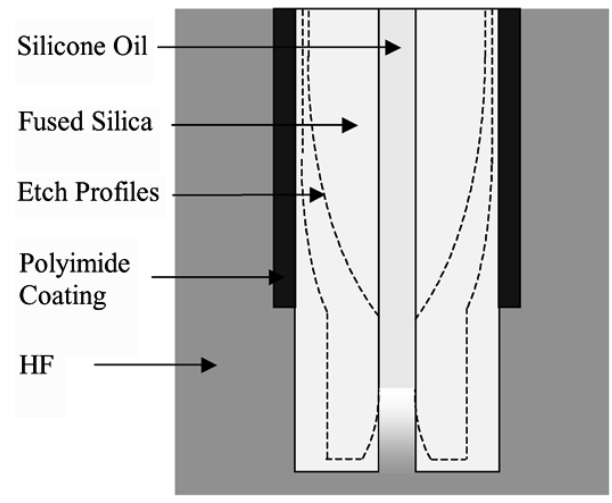

Fig. 8. Schematic of the diffusion-limited sharpening process.

from [11]. The first order reaction kinetic coefficient is calculated by scaling the bulk-etching rate $\left(1.5 \mu \mathrm{m} \mathrm{min}^{-1}\right)$ with the surface area to volume ratio of the etching condition. The effective diffusion length is then estimated to be $143 \mu \mathrm{m}$, which is in reasonable agreement with our experimentally determined value.

\section{B. Sharpened Microinjector}

Microinjectors with highly tapered tips can be fabricated by utilizing the aforementioned diffusion limited etching process. The polyimide coating is partially removed and the capillary is immersed such that the entire bare silica portion is in etchant, as shown in Fig. 8. The polyimide coating is permeable to HF molecules. This was confirmed with two experiments. In the first, a capillary whose ends were sealed with baked photoresist was fully immersed in an etchant solution. In a second experiment, the capillaries were bent into a closed loop and only partially immersed in HF solution, carefully keeping the ends outside the etchant bath, thereby eliminating the possibility that HF would seep through the polyimide coating. The two experiments consistently showed that the wetted portion of the capillary underneath the polyimide coating was etched. The etching rate was experimentally determined to be roughly $0.08 \mu \mathrm{m} \mathrm{min}^{-1}$. Since HF molecules can diffuse across the polyimide coating and etch the capillary, a small gap is formed between the polyimide coating and the silica capillary after initiation of the etching process. At this point, HF molecules can diffuse into the gap from the open end of the polyimide

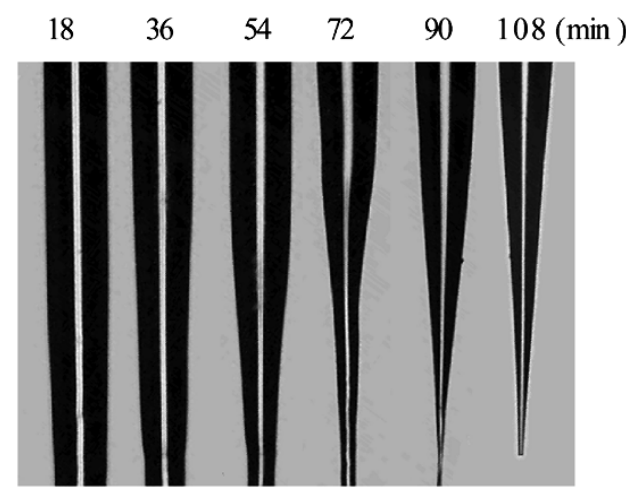

Fig. 9. Capillaries at intermediate etching stages during tip formation. The outer diameter of the capillary before etching is $325 \mu \mathrm{m}$. The polyimide coating was removed after the etching process to take the pictures.

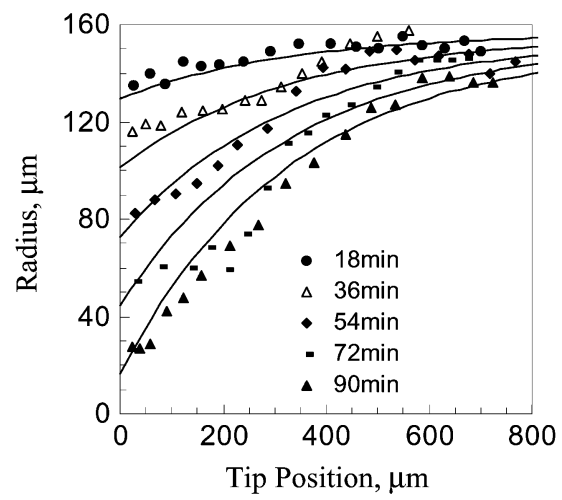

Fig. 10. Profiles of the microinjector as a function of etching time. Solid lines represent the etched profiles calculated by the diffusion model.

coating. The etching mechanism is the same as the 1-D diffusion model developed for the single-cell trapper formation. Fig. 9 shows pictures of the capillary at intermediate stages of the sharpening process. This technique allows for the fabrication of much longer tips than in the previous case. Fig. 10 shows the experimentally determined tip profiles at different etching times. These results are compared with a model that considers both diffusion and etching across the coating. A good correlation has been obtained between the etched profiles and the diffusion model.

\section{Discussion}

We have described the fabrication of microsurgical tools obtained by chemical etching of microcapillaries. To provide a more accurate description of the etching processes, other effects such as higher order reaction kinetics, transient dynamics, and microconvection should be considered. Transient dynamics and high order reaction kinetics have shown to be important in the etching of high aspect ratio sacrificial layers, where the material-etchant interface is changing with time [11]. Microconvection is believed to be the etching mechanism for the sharpening of chalcogenide fibers in piranha solution [12]. The local distribution of etchant concentration because of the microconvection pattern provides a reasonable explanation of the necking effect observed in their experiment. However, microconvection is unlikely to be important in our etching condition since we did not observe any necking effect. Furthermore, the high axial etching 


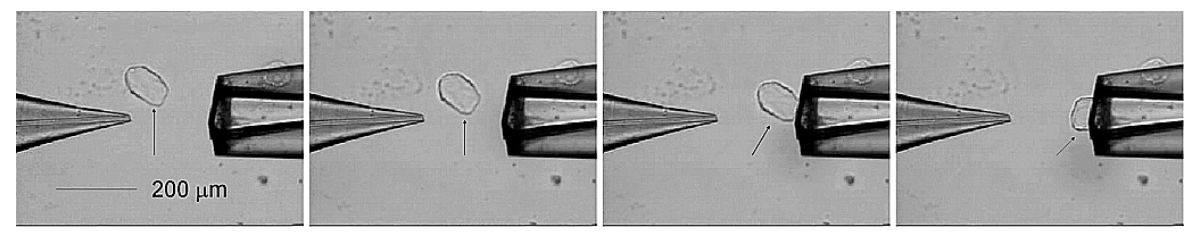

Fig. 11. Video time series showing the capture of a cabbage cell with the single-cell trapper. The images are separated by $1.5 \mathrm{~s}$.

rate in the highly tapered tip etching cannot be explained by microconvection. As shown in Fig. 9, the tapered length can be longer than $2 \mathrm{~mm}$ for an etching time of $108 \mathrm{~min}$, which is equivalent to an etch rate of $\sim 20 \mu \mathrm{m} \mathrm{min}^{-1}$. This is much higher than the measured bulk-etching rate of $1.5 \mu \mathrm{m} \mathrm{min}^{-1}$. The difference in the etching mechanisms is likely due to the different materials and etchant used in the experiments.

Formation of optical fiber tips inside polymer coatings has previously been described by other research groups [13], [14]. The process is referred to as tube-etching, which produces tips with a smooth surface for scanning near-field optical microscopy. For an impermeable coating, microconvection is proposed as the sharpening mechanism inside the polymer coating [14]. For a permeable coating, sharpening is suggested to be a result of a gradient in the lateral diffusion along the tip in the meniscus region [14]. In our case, the polyimide coating is found to be slightly permeable to HF. However, the etching rate of fused silica across a polyimide coating is only $0.08 \mu \mathrm{m} \mathrm{min}{ }^{-1}$, which is much smaller than the bulk-etching rate of $1.5 \mu \mathrm{m} \mathrm{min}^{-1}$. A much longer etching time would be required for the tip formation than the $108 \mathrm{~min}$ in our experiment. Moreover, taper is always observed near the polyimide-coated end rather than the region of the etchant-solvent interface. Therefore, lateral diffusion gradient is unlikely to be important in our etching condition. The difference between our experiment and tubing etching could be a result of the different permeabilities of the polymer coatings used in the experiments. Further investigation is required to clarify this point.

The present diffusion-reaction model already covers the major features of the etching processes. Despite the simplifications adopted, the model correlates well with the etch profiles of both the single-cell trapper and the highly tapered microinjector. This suggests that the etching dynamics are dominated by the proposed mechanism for our etching conditions. The model provides a fundamental understanding of the process and offers insights for modification of the device geometry. Based on the diffusion model, single-cell trappers with tailored dimensions can be fabricated by simply controlling the etch time. Moreover, the trapper's inner geometry and the injector tip profiles can be adjusted by modifying the effective diffusion length, which changes the steady state concentration distribution of the etchant. The effective diffusion length can be modified by controlling the etching temperature or the etchant used.

\section{INTRACYTOPLASMIC INJECTION}

The utility of the micromanipulators is demonstrated by an intracytoplasmic injection experiment. Intracytoplasmic injection is a powerful tool in genetics [15], assisted fertilization [7],
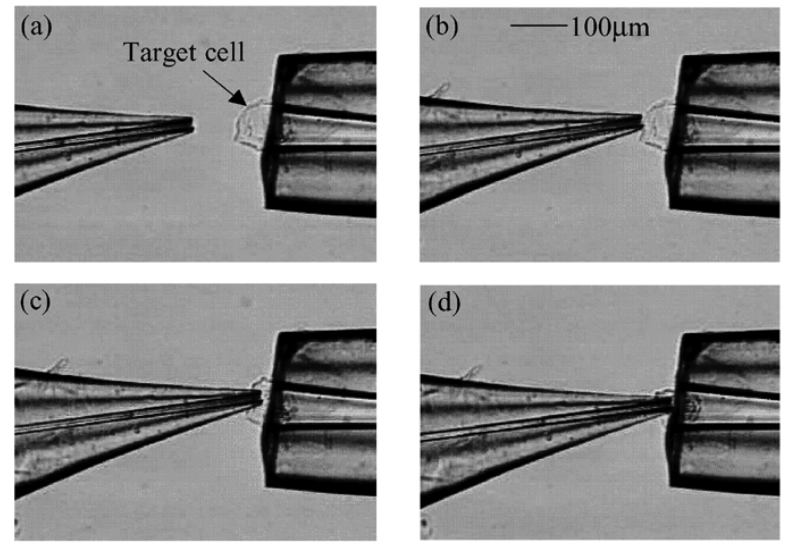

Fig. 12. Video images demonstrating intracytoplasmic injection (see also text).

and other cellular research [16]. In our experiments, metabolically competent brassica oleracea (cabbage) protoplasts that are roughly $50 \mu \mathrm{m}$ in diameter are used. The cells are prepared by cutting fresh cabbage into segments of roughly $1 \mathrm{~mm}$ length. The segments are then incubated with a digestion medium containing $500 \mathrm{mM}$ D-Sorbitol (Sigma S-1876), $1 \mathrm{mM} \mathrm{CaCl} 2$ (Sigma, 23922-4), 5 mM MES (Sigma, M-8250), 2\% cellulase (Sigma, C-9422), and $0.3 \%$ pectinase (Sigma, P-2401). The $\mathrm{pH}$ value is adjusted to 5.5 with the addition of $\mathrm{KOH}$. After incubation for 3 hours at room temperature, the cells are washed out by gently shaking the tissue within an isotonic medium containing $500 \mathrm{mM}$ D-Sorbitol, $1 \mathrm{mM} \mathrm{CaCl}_{2}$, and $5 \mathrm{mM} \mathrm{MES}$. The $\mathrm{pH}$ value of the medium is adjusted to 6 .

The intracytoplasmic injection experiments are performed on an inverted microscope (Nikon Eclipse TE200) for phase contrast and epifluorescence microscopy. The single cell trapper is kept in a fixed location, while the position of the microinjector is controlled by a translation stage mounted on the microscope. Extra care should be taken to prevent breakage of the sharp tip. Both micromanipulators are connected to digital syringe pumps (Harvard apparatus 44I/W). Fig. 11 shows a video time series of a cell captured with the single-cell trapper. Adjusting the internal pressure of the single cell trapper generates a flow field around the trapper. The hydrodynamic suction force produced on the target cell in the flow field is sufficient to drag the cell to the trapping site. Then, the translation stage advances the injector toward the trapper until the cell membrane is penetrated. Fig. 12 shows video images of the cell membrane penetration with a microinjector. After the single cell trapper captures the target cell [see Fig. 12(a)], the microinjector is moved toward the cell and placed in contact with the cell membrane [see Fig. 12(b)]. The cell membrane deforms under the force applied by the tip of the microinjector [see Fig. 12(c)]. Finally, 

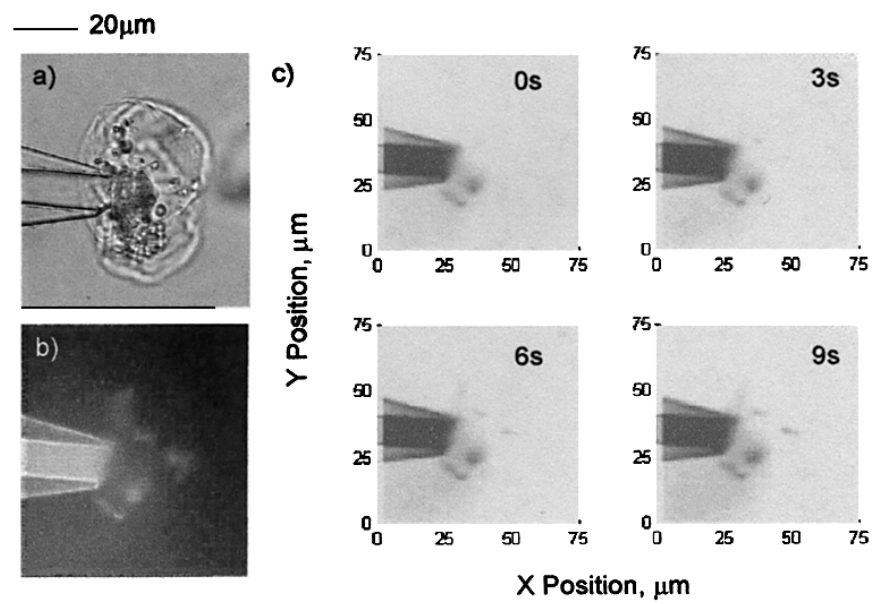

Fig. 13. (a) Phase contrast image of a cell penetrated by a microinjector, (b) fluorescence image of the same cell, and (c) intensity contours illustrating accumulation of the dye.

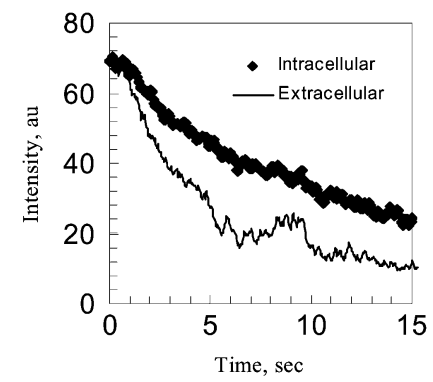

Fig. 14. Comparison of intracellular and extracellular photobleaching.

the tip breaks though the membrane and penetrates the cell [see Fig. 12(d)].

With the capability of cell membrane penetration, a variety of molecules can be directly delivered into a target cell by means of the microinjector. A fluorescent dye, rhodamine B, is injected into a cell to demonstrate fluid delivery. Fig. 13(a) and (b) shows the phase contrast and fluorescence images of the cell. Rhodamine B selectively accumulates in some regions inside the cell [see Fig. 13(c)]. The dye binds selectively to some organelles and accumulates in these locations. Furthermore, we can measure photobleaching within the cell. Photobleaching is the decay of fluorescence intensity due to photochemical reactions of the dye molecules [see Fig. 14]. Measurement of photobleaching allows direct probing of the intracellular physiological condition, since the photobleaching rate depends on several physiological factors, such as oxygen concentration [17].

\section{CONCLUSION}

We have developed simple and accurate processes based on chemical etching techniques to fabricate microsurgical tools for single cell trapping and intracytoplasmic injection. The simplicity and reproducibility of the chemical etching processes can be easily adopted for low cost mass production. Furthermore, the ability to precisely control etch profiles combined with the wide range of dimensions for commercially available capillary tubing creates opportunities to fabricate microsurgical tools for various biological applications. Micromanipulators fabricated from capillaries provide an effective way to manipulate bioparticles at the cellular level. With precise fluidic control, it is possible to deliver fluids with a precise quantity of molecules into target cells. This allows direct interaction with the complex cellular system at a molecular level.

\section{ACKNOWLEDGMENT}

The authors would like to thank Prof. T.-H. Wang for his valuable discussion and W. Tan for her technical assistance.

\section{REFERENCES}

[1] C.-J. Kim, A. P. Pisano, and R. S. Muller, "Silicon-processed overhanging microgripper," J. Microelectromech. Syst., vol. 1, pp. 31-36, 1992.

[2] Y. Lu and C.-J. Kim, "Micro-finger articulation by pneumatic parylene balloons," in Proc. Transducers'03, 2003, pp. 276-279.

[3] H. Y. Chan and W. J. Li, "A thermally actuated polymer micro robotic gripper for manipulation of biological cells," in Proc. IEEE ICRA 2003, 2003, pp. 288-293.

[4] C.-M. Ho, "Fluidics-The link between micro and nano science and technologies," in Proc. IEEE MEMS Workshop, 2001, pp. 375-384.

[5] P. K. Wong, Y.-K. Lee, and C.-M. Ho, "Deformation of DNA molecules by hydrodynamic focusing," J. Fluid Mech., vol. 497, pp. 55-65, 2003.

[6] A. C. F. Perry, T. Wakayama, H. Kishikawa, T. Kasai, M. Okabe, Y. Toyoda, and R. Yanagimachi, "Mammalian transgenesis by intracytoplasmic sperm injection," Science, vol. 284, pp. 1180-1183, 1999.

[7] J. Tesarik and C. Mendoza, "In vitro fertilization by intracytoplasmic sperm injection," BioEssays, vol. 21, pp. 791-801, 1999.

[8] D. R. Turner, "Etch Procedure for Optical Fibers," U.S. Patent 4469554, Sept. 4, 1983.

[9] P. K. Wong, T.-H. Wang, and C.-M. Ho, "Optical fiber tip fabricated by surface tension controlled etching," in Proc. of Hilton Head, 2002, pp. 431-434.

[10] F. W. Sears, M. W. Zemansky, and H. D. Young, University Physics, 7th ed. Reading, MA: Addison-Wesley, 1987.

[11] J. Liu, Y.-C. Tai, J. Lee, K.-C. Pong, Y. Zohar, and C.-M. Ho, "In situ monitoring and universal modeling of sacrificial PSG etching using hydrofluoric acid," in Proc. IEEE MEMS Workshop, 1993, pp. 71-76.

[12] M. A. Unger, D. A. Kossakovski, R. Kongovi, J. L. Beauchamp, J. D. Baldeschwieler, and D. V. Palanker, "Etch chalcogenide fibers for near-field infrared scanning microscopy," Rev. Sci. Instrum., vol. 69, pp. 2988-2993, 1998.

[13] P. Lambelet, A. Sayah, M. Pfeffer, C. Philipona, and F. Marquis-Weible, "Chemically etched fiber tips for near-field optical microscopy: A process for smoother tips," Appl. Opt., vol. 37, pp. 7289-7292, 1998.

[14] R. Stöckle, C. Fokas, V. Deckert, R. Zenobi, B. Sick, B. Hecht, and U. R. Wild, "High-quality near-field optical probes by tube-etching," Appl. Phys. Lett., vol. 75, pp. 160-162, 1999.

[15] J. W. Gordon, G. A. Scangos, D. J. Plotkin, J. A. Barbosa, and F. H. Ruddle, "Genetic transformation of mouse embryos by microinjection of purified DNA," in Proc. Nat. Acad. Sci. USA, vol. 77, 1980, pp. $7380-7384$.

[16] J. Perlette and W. Tan, "Real-time monitoring of intracellular mRNA hybridization inside single living cells," Anal. Chem., vol. 73, pp. 5544-5550, 2001.

[17] P. K. Wong, L. J. Chun, and C.-M. Ho, "Multi-color single filter photobleaching analysis for measuring gene expression," in Proc. 2nd Joint Meeting of EMBS-BMES, vol. 1, 2002, pp. 629-630.

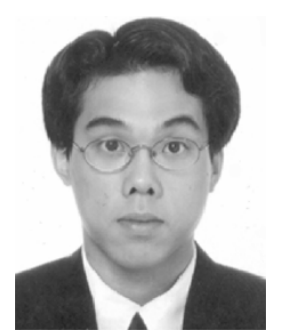

Pak Kin Wong received the B.Eng. degree in mechanical and automation engineering (renamed Department of Automation and Computer-Aided Engineering in summer 2000) from The Chinese University of Hong Kong and the M.S. degree in mechanical and aerospace engineering from the University of California at Los Angeles (UCLA) in 1999 and 2001, respectively. He is currently a doctoral candidate in the MEMS program at UCLA, where he is developing various molecular manipulation techniques for bio- and nanotechnology. 


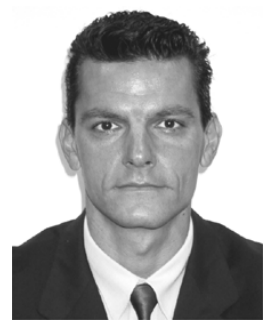

Umberto Ulmanella received the Laurea degree in mechanical engineering from the Universitá di Padova, Italy, in 1995 and the M.S. degree in mechanical and aerospace engineering from the University of California at Los Angeles (UCLA) in 1998 . He is currently a doctoral candidate in the Department of Bioengineering at UCLA, where he has been working on micro- and nanochannel flow.

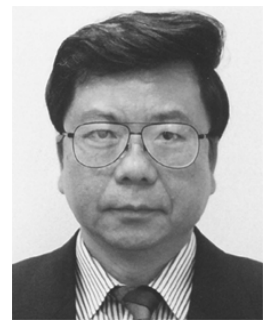

Chih-Ming Ho (M'00) received the Ph.D. degree in mechanics from The John Hopkins University, Baltimore, MD, in 1974.

He currently holds the Ben Rich-Lockheed Martin Professor in the Henry Samueli School of Engineering and serves as UCLA Associate Vice Chancellor for Research. He is the Director of the Institute for Cell Mimetic Space Exploration (CMISE). In 1997, he was inducted as a member of the National Academy of Engineering and in 1998, Sinica.

Dr. Ho is a Fellow of the American Physical Society as well as American Institute of Aeronautics and Astronautics for his contributions in a wide spectrum of technical areas. 